\title{
REVIEW
}

\section{QT dispersion}

\author{
P D Higham, R W F Campbell
}

Ventricular tachyarrhythmias can be dangerous. They may result from cardiovascular disease, principally ischaemic heart disease, or they may be an unwanted effect of drug treatment. The surface electrocardiogram has been investigated for its ability to predict either the occurrence of these arrhythmias or their clinical outcome, sudden death. Holter monitoring seemed the most promising of these electrocardiographic techniques with analysis of the frequency and timing of extrasystoles and more recently of heart rate variability. Signal averaging also contributes by identifying abnormalities of QRS duration and configuration. These techniques are useful but they are neither sensitive nor specific. They are applied to conduction abnormalities, autonomic disturbances, and trigger phenomena but they provide no information on what may be the most important arrhythmogenic factor-the heart's recovery of ventricular excitability. Currently, the only routine assessment of ventricular recovery is the traditional measurement of a single QT interval. This measurement is not standardised. Recommendations for QT measurement have not defined a specific lead(s), have suggested a single defined lead (lead II or the lead with the largest $T$ wave), or have proposed a mean QT derived from an arbitrary subset of leads. This lack of a systematic approach may explain the variation in sensitivity and specificity claimed for single lead measurement of QT interval in predicting life threatening ventricular arrhythmias. The presence of interlead QT variation together with the demonstration that in healthy individuals the longest QT interval is most frequent in leads V2 to $\mathrm{V}^{1}$ and that the QT interval is not closely related to $T$ wave heigh $t^{2}$ challenges the assessment of ventricular recovery by measurement of a single QT interval.

QT dispersion addresses this challenge. We have defined QT dispersion as QT maximum minus QT minimum as measured from the 12 lead electrocardiogram (figure). If necessary, and within the limitation of the technique, values can be rate corrected. QT dispersion is a measure of interlead QT variability, a phenomenon described by Campbell and coworkers, ${ }^{1}$ who demonstrated small but consistent differences between the QT intervals of different leads measured by a digitiser program. Increasing evidence sug- gests that the presence and degree of interlead QT variability, far from being a recording artefact, may provide clinically valuable information by reflecting underlying disturbances of ventricular recovery. ${ }^{3}$

\section{QT dispersion after myocardial infarction} The clinical and research potential of QT dispersion (and other subtle aspects of the QT interval) has yet to be fully established but evidence from studies after myocardial infarction and from its use in the assessment of antiarrhythmic drug treatment suggest an important role. In 1985 Mirvis, using body surface mapping, first showed considerable regional differences in QT intervals over the chest wall in patients 24 hours after acute myocardial infarction compared with controls. ${ }^{4}$ Cowan et al confirmed the increased dispersion of the surface 12 lead QT interval in patients with one day old myocardial infarction (anterior infarcts, mean (SD) 70 (30) $\mathrm{ms}$; inferior infarcts, 73 (32) $\mathrm{ms}$ ) compared with a group of patients without cardiac disease (48 (18) $\mathrm{ms}$ ) and suggested that this increase in dispersion reflected underlying dispersion of repolarisation caused by infarction. ${ }^{2}$ New data suggest that QT dispersion is greatest in the early hours of infarction, falls with time and successful thrombolysis, and may be highest in patients in whom ventricular fibrillation develops. ${ }^{56}$

\section{QT dispersion and assessment of} antiarrhythmic drug treatment

QT dispersion changes after acute myocardial infarction are interesting, but as yet they have no clinical application. In the context of antiarrhythmic drug efficacy and safety, however, they are already useful. Day et al found increased QT dispersion in arrhythmia patients with long QT intervals but normal or reduced levels of dispersion in patients with long QT intervals who were taking sotalol.? No patient in the sotalol group had a QT dispersion greater than $100 \mathrm{~ms}$, whereas only one patient in the arrhythmogenic group had a QT dispersion result of less than $100 \mathrm{~ms}$. Most of the arrhythmia patients had the long QT syndrome, though in one the arrhythmias were secondary to quinidine toxicity. Linker 


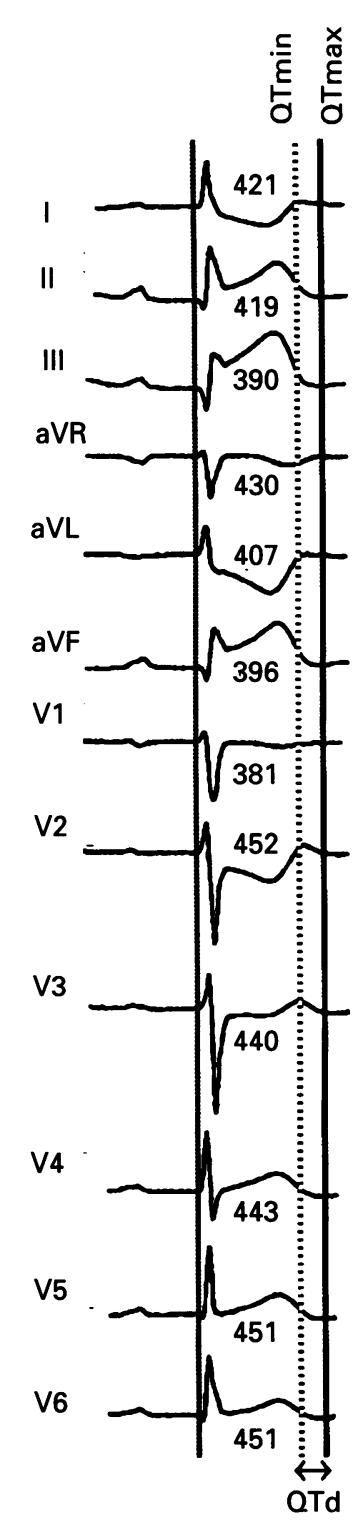

Electrocardiogram from a patient with acute inferior infarction showing dispersion of the $Q T$ intervals. $Q T$ dispersion $(Q T d)=Q T \max (V 2)$ minus $O T \min (V 1)$. In this example QTd was $71 \mathrm{~ms}$. et al showed levels of QT dispersion similar to those reported by Day et al in nine patients with the long QT syndrome. Oral $\beta$ blockade controlled symptoms in five of these patients but the treatment did not affect QT dispersion. ${ }^{8}$ Such a result might be expected because uniform blockade of sympathetic tone may modulate the arrhythmia substrate but is unlikely to produce regional changes in ventricular recovery. Drugs with class III antiarrhythmic actions can produce regional changes in ventricular recovery. Day and coworkers showed a reduction in QT dispersion in patients taking sotalol after myocardial infarction. ${ }^{9}$ Very recently Hii and coworkers showed an increase in the precordial dispersion of the QT interval in patients receiving class Ia antiarrhythmic drugs who had torsades de pointes. The same patients when subsequently treated with amiodarone without recurrence of their arrhythmia but with similar QT interval prolongation showed no increase in QT dispersion. ${ }^{10}$ Thus QT dispersion may predict not only antiarrhythmic drug efficacy but also, and more importantly, arrhythmogenesis.

\section{Theoretical considerations}

The surface electrocardiographic QT interval reflects complex and interrelated aspects of cardiac electrophysiology, cardiac geometry, torso shape, tissue impedance, and biological signal processing. Mathematical and physical models can recreate the QRS complex from measurement of myocardial potentials. Were these models able to explain the generation of the $T$ wave then they would help to identify factors that are crucial to the body surface expression of QT dispersion. While regional changes in action potential duration and conduction will play an important part, other abnormalities that occur in a non-uniform distribution within the myocardium and in a sufficient volume to be detectable at the body surface may well alter QT dispersion. These may be electrophysiological-for example, local populations of cells with afterdepolarisations-geometric, as would occur with regional wall motion abnormalities, or asymmetrical hypertrophy; or neurohumoral, as when there are regional alterations in autonomic tone.

\section{Methodological considerations}

Currently QT dispersion is measured by digitisers, ${ }^{1}$ photocopy enlargement, ${ }^{10}$ and by the use of digital computing systems. ${ }^{11}$ It is too early to accept any of these as the reference standard for clinical use. For the present, the most crucial aspect of methodology is the protocol to define the end of the $T$ wave. This definition is especially difficult when $T$ waves merge with $U$ or $P$ waves, so we have defined the end of the $T$ wave as the point of return to the TP baseline or the nadir between the $T$ and $U$ wave. In our research we have always used this protocol-if we have been making errors in identifying the end of the $T$ wave then we have made consistent errors and none the less we have shown important electrophysiological associations. We believe that the measurement protocol must allow an observer to omit measurement in a lead where there is uncertainty. To force a QT measurement seems likely to create an outlier measurement that may be a spurious QT max or QT min. None the less it is important to evaluate all 12 leads for measurement. The digitiser is a labour intensive instrument but has the advantage of allowing the input of measured points without bias from previous measurements. Each point is separate. No immediate readout is shown and the sequence of measurements means that it is all but impossible to "line up" the QT intervals across leads. The precision of these methods varies as do the normal values quoted. There is a small but significant interobserver variation in the measurement of QT dispersion. From our own experience the standard deviation of repeat measurements is approximately $6 \mathrm{~ms}$ when an experienced observer measures from recordings made at $50 \mathrm{~mm} / \mathrm{s}$ and $10 \mathrm{mV} / \mathrm{cm}$. When the digitiser is used the normal rate-corrected values for QT dispersion are between $20 \mathrm{~ms}$ and $50 \mathrm{~ms}$ with values after infarction rising to $60-100$ $\mathrm{ms}$ and to as high as $150-200 \mathrm{~ms}$ in patients with long QT syndromes.

Traditional electrocardiographic lead positions owe little to science. They were chosen initially for technical convenience and then to document patterns of activation. They may not necessarily be the best lead positions to reflect the process of recovery of ventricular excitability. When such exciting results have been obtained from standard electrocardiogram leads it seems possible that more information, greater reliability, and easier measurement may come from exploring other signal sources. QT interval changes are dynamic and for this reason better information is likely to come from serial measurements within patients (for example, to document the effect of disease progression or to assess the effects of drug treatment) rather than by using single static values. Improved algorithms for accurate measurement of the QT end point should lead to the development of continuous monitoring systems enabling minute to minute fluctuations or even beat to beat evaluation in patients in coronary care units or intensive therapy units.

\section{Conclusions}

Interlead QT variation and its measurement as QT dispersion challenges our current approaches to the electrocardiographic assessment of arrhythmia risk. QT dispersion provides a potentially simple, cheap, noninvasive method of measuring underlying dispersion of recovery of ventricular excitability. With continued development QT dispersion may provide important clinical benefits, particularly in the assessment of the benefits and risks of antiarrhythmic drug treatment. 
1 Campbell RWF, Gardiner P, Amos PA, Chadwick D, Jordan RS. Measurement of the QT interval. Eur Heart f 1985;6(suppl D):81-5.

2 Cowan JC, Yusoff K, Moore $M$, et al. Importance of lead selection in QT interval measurement. $A m \mathcal{F}$ Cardiol 1988;61:83-7.

3 Higham PD, Hilton CJ, Aitcheson DA, Furniss SS, Bourke JP, Campbell RWF. QT dispersion does reflect regional variation in ventricular recovery. Circulation 1992;86(suppl I):392.

4 Mirvis DM. Spatial variation of the QT intervals in normal persons and patients with acute myocardial infarction. $\mathcal{A}$ Am Coll Cardiol 1985;5:625-31.

5 Higham PD, Furniss SS, Campbell RWF. Increased OT dispersion in patients with ventricular fibrillation followin

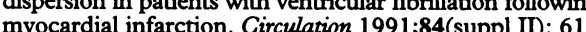
myocardial infarction. Circulation 1991;84(suppl II): 61. Heperfusion, acute myocardial infarction Reperfusion, acute myocardial infarction and
dispersion [abstr]. Eur Heart $\mathcal{f}$ 1992;13(suppl):448.
7 Day CP, McComb JM, Campbell RWF. QT dispersion an indicator of arrhythmia risk in patients with long QT intervals. Br Heart $\mathcal{F} 1990 ; 63: 342-4$.

8 Linker NJ, Colonna P, Kekwick CA, Till J, Camm AJ, Ward DE. Assessment of QT dispersion in patients with congenital long QT syndromes. Am f Cardiol 1992; 69:634-8.

9 Day CP, McComb JM, Matthews J, Campbell RWF Reduction in QT dispersion by sotalol following myocardial infarction. Eur Heart f 1991;12:423-7.

10 Hii JTY, Wyse DG, Gillis AM, Duff HJ, Solyo MA Mitchell LB. Precordial QT interval dispersion as a marker of torsades de pointes. Disparate effects of class marker of torsades de pointes. Disparate effects of class 1992;86:1376-82.

11 de Bono DP, Bhullar HK, Goddard WP. Automated measurement of QT dispersion identifies patients at risk measurement of QT dispersion identifies patients at risk
from ventricular tachycardia [abstr]. Eur Heart $\mathcal{f} 1992$; 13:369.

\section{Commentary}

\section{Is this the end of the aspirin and vein graft story?}

Fourteen years and at least 20 randomised studies after the issue was first raised, antiplatelet therapy after coronary artery bypass grafting remains a subject for research trials, with apparent uncertainty among clinicians about the applicability of the various trials to clinical practice. van der Meer et al investigated whether aspirin or warfarin should be used, and if aspirin at what doses, when should it be started, and for how long it should be continued. ${ }^{1}$ During grafting the endothelium of the saphenous vein is damaged by harvesting and during insertion into the arterial system. Subsequent platelet adhesion to exposed subendothelial tissue causes thrombus formation. Approximately $10 \%$ of vein grafts occlude within the first three months, and a further $10 \%$ are lost by one year, with $2 \cdot 5-5 \%$ a year being lost thereafter. The antiplatelet agents so far tested in trials are being used as antithrombotic agents because many of them were used to reduce the early failure rate, thought due to thrombotic occlusion, rather than the late (greater than one year) loss, which was thought to be caused by the development of intimal hyperplasia.

Results from the various trials may be difficult to compare because they are confounded by variables such as the timing of the start of treatment, the dose given, its mode of administration, whether grafts or anastomoses are being counted, the diameter of the grafted artery, the adequacy of its run off, and surgical consistency (early thrombotic occlusion may be influenced to some degree by technical factors including whether or not endarterectomy was undertaken). Information on which agent should be given, when it should be started, and in what dose can, however, be summarised. The choices of regimens seem to be-aspirin alone, aspirin plus dipyridamole, or warfarin. Several trials showed that aspirin in a dose of $325 \mathrm{mg}$ once a day is as good as aspirin plus dipyridamole, though when lower doses of aspirin were evaluated additional dipyridamole seemed to confer an advantage. Doses greater than $325 \mathrm{mg}$ once a day or of $325 \mathrm{mg}$ given more than daily increased gastrointestinal side effects with no added efficacy. The lack of a placebo group in the Dutch trial in which $50 \mathrm{mg}$ of aspirin was used and other ex vivo evidence of reduced efficacy of such a dose on platelet thromboxane $A_{2}$ inhibition mean that there is still no reliable evidence to indicate that any dose other than $325 \mathrm{mg}$ is appropriate.

\section{What of warfarin?}

Several trials, including that of van der Meer et al showed that aspirin is as good as warfarin. This is perhaps not surprising because aspirin, in preventing early occlusion, is being used as an antithrombotic agent. Because of its association with increased morbidity and the need for regular testing of the international normalised ratio warfarin is less and less used.

\section{When should aspirin be started?}

There has been considerable argument about the best time to start treatment. Ideally aspirin should be present when interaction between the damaged vessel wall and blood starts; this suggests that it should be given preoperatively. Indeed a meta-analysis of 13 randomised trials confirmed that greater efficacy is seen with earlier treatment. However, studies have shown that aspirin given as a single preoperative dose was no better than when it was given 6 hours after the procedure. Whereas aspirin given earlier did not seem to increase blood loss there was a non-significant but worrying increase in the reoperation rate $(4 \cdot 8 \%$ v $1 \cdot 0 \%)$. Aspirin can be given within 6 hours via a nasogastric tube or as a suppository.

\section{How long should aspirin be continued?}

In trials of its influence on graft patency aspirin was of confirmed benefit in the first year, especially by reducing thrombotic events in high risk grafts. The current view is that aspirin should be continued indefinitely, but the benefits of long-term aspirin have not been established.

Thus evidence from published trials suggests that the best available treatment to prevent thrombotic occlusion of vein grafts is aspirin given as sole agent in a dose of $325 \mathrm{mg}$ by mouth, within 6 hours of the operation and continued for at least one year. Even so, up to $7 \%$ of grafts may still occlude early, and late failure remains a problem. The challenge is to reduce the incidence of early thrombosis further while maintaining systemic haemostasis and also to promote long-term vein graft patency by inhibiting intimal hyperplasia. This process continues to occur after angioplasty despite nearly fifty trials of prevention in the past six years.

Division of Cardiology,

A H GERSHLICK

Department of Medicine,

Glenfield General Hospital,

Leicester $L E 39 Q P$.

1 van der Meer J, Hillege HL, Kootstra GJ, Ascoop CAPL, Pfisterer M, van Gilst WH, Lie KI, for the CABADAS research group of the Gilst WH, Lie KI, for the CABADAS research group of the Interuniversity Cardiology Institute of the Netherlands. Prevention of one-year vein-graft occlusion after aortocoronary bypass surgery: a comparison of low-dose aspirin, low-dose aspirin
oral anticoagulants. Lancet 1993;342:257-63. 